Learning from serious incidents

\section{Will the future continue to repeat the past?}

\section{S J Woodward}

\section{How organisations can learn from the experience of incidents to prevent future harm}

A cross different healthcare systems worldwide, published reports of inquiries into serious incidents urge that the lessons that emerge must be learnt and that such incidents should not happen again. But they do. The lessons, it seems, are rarely learnt and the perception is that health services are not trying hard enough-if at all. ${ }^{12}$ While it might appear straightforward, in reality not only are the reasons for the incident usually multifactorial, but implementing change is fraught with difficulties. This should not be taken as an excuse, but as a stimulus to understand why it is difficult to learn lessons and how the experience of incidents can be used by others to prevent future harm.

One of the fundamental barriers to learning lessons is the lack of a safety culture in health care. A difficult but essential aspect of health care is the need to accept that people, processes, and equipment in highly complex systems will fail. While the great majority of treatment is carried out effectively and safely, all organisations will at one time or another experience a serious incident. By accepting this, people and organisations can then focus on learning and making changes which seek to design out opportunity for error or failure and develop defences and contingency plans to cope with these failures. ${ }^{3}$

However, the all too common response to a serious incident is to focus on individual frontline workers who may then face disciplinary measures, suspension from work, and subsequent professional censure. ${ }^{4}$ A good safety culture, therefore, is one where an organisation promotes active awareness, where staff are encouraged to speak up and identify conditions and practices that might lead to an incident, and where staff-when they do speak upare treated fairly.

Furthermore, there is a tendency to concentrate on immediate causes such as human error rather than on organisational causes which are prevalent in many incidents. ${ }^{5}$ While the immediate causes of an accident are important, and putting these right may prevent the same incident from happening again, making changes to the underlying or latent causes is more likely to prevent future incidents. ${ }^{6}$ By ensuring that incidents are seen as systems failures it is possible for departments - and even organisations-other than those involved in the incident to learn far more than would first appear possible. ${ }^{5}$ This could be achieved by investigating incidents using a consistent methodology across healthcare settings and sharing the relevant lessons to enable wider learning.

Lessons learnt following incidents are all too often confined to those directly involved rather than disseminated throughout the health service. ${ }^{5}$ Most serious incidents repeat past events, but the learning from previous incidents is usually at an individual or, at best, department level and individuals then leave the organisation taking their knowledge with them, depleting the organisation of its memory. ${ }^{7}$ A further challenge to spreading change across health services is that, unless an individual or team is directly involved in an incident, they may feel it could not happen to them and therefore that any change or learning required does not apply. In addition, even when people feel they can speak up, they may not believe that this will make a difference to the system within which they work. ${ }^{8}$ Organisations often fail to show the benefits of reporting and to demonstrate how staff can influence the organisational levels of risk or change the system.

Another problem is that learning is not sustained: an organisation may focus intensively on a problem for a short time but is distracted when new priorities emerge or staff move on. Organisations need to ensure that they set up knowledge and risk management systems for sharing lessons on an ongoing basis. To retain a corporate memory all incident investigation records must be retained and the recommendations made supported by implementation plans. A final report should clearly describe the incident, the causal analysis, and proposed recommendations for change. Many investigators make the mistake of producing an unfeasibly large number of recommendations-itself a barrier to learning-or they provide recommendations which staff can do little about and lack clarity on who is responsible for implementation. Reports should have key recommendations which are prioritised, realistic, and sustainable. These should be translated into implementation plans which are monitored until completion and evaluated for impact. These reports are then used to disseminate the learning to help others.

It is also important to note that serious incidents should not be the only catalyst for change. Organisations should take as much notice of incidents that were prevented or which caused little or no harm. These provide vital information on where a system is failing or likely to fail. If tackled early, the changes made could prevent a more serious incident occurring. ${ }^{9}$ Consequently, incident reporting systems need to be quick and easy to use, and enable staff to report all levels of incidents in sufficient detail to enable appropriate analysis. There is a need to prioritise incidents for further detailed investigation and, if the analysis demonstrates significant themes and clusters of incidents in relation to specific factors, resources should be targeted at the areas that require appropriate changes. Feedback to all staff to demonstrate these changes is essential to re-enforce the benefits of reporting.

The effects of serious incidents for patients, their relatives, and healthcare staff are long lasting. We therefore owe it to our patients and our staff to have an effective system that ensures that the lessons learned are long lasting. If we put into place the processes suggested, we have a realistic and sustainable chance of ensuring that experience of one incident can prevent future harm.

Qual Saf Health Care 2005; 14:74.

doi: 10.1136/qshc.2005.013722

Correspondence to: Ms S J Woodward, National Patient Safety Agency, 4-8 Maple Street, London, WIT 5HD; suzette.woodward@ npsa.nhs.uk

\section{REFERENCES}

1 Department of Health. An organisation with a memory. London: Stationery Office, 2001

2 Institute of Medicine. To err is human: building a safer health system. Washington DC: National Academy Press, 2000.

3 Reason J, Hobbs A. Managing maintenance error. A practical guide. Hampshire, UK: Ashgate Publishing Company, 2003.

4 Reason J. Human error: models and management. BMJ 2000;320:768-70.

5 Toft B, Reynolds S. Learning from disasters: a management approach. Oxford: ButterworthHeinemann, 1994

6 Kletz T. Still going wrong! Case histories of process plan disasters and how they could have been avoided. Oxford: Butterworth-Heinemann, 2004.

7 Kletz T. Lessons from disaster - how organisations have no memory and accidents recur. Institution of Chemical Engineers, 1993.

8 Reason J. Beyond the organisational accident: the need for "error wisdom" on the frontline. Qual Saf Health Care 2004; 13(Suppl II):ii28-33.

9 National Patient Safety Agency. Seven steps to patient safety, 2004. http://www.npsa.nhs.uk (accessed 20 February 2005). 


\section{Can quality improvement be used to change the wider healthcare system?} N Edwards

$\mathrm{T}$ he quality movement has made major strides in developing a discipline and methodology for improvement, mostly focused on the front line of healthcare delivery. The planning of the level above this-hospitals and other large systems that deliver health services does not have the same rigour or discipline. Yet, the results of poor design of a hospital or of processes linking parts of health care together can be just as serious for patients as an unsafe clinical procedure. The lack of methodology also matters because the need to redesign the way that health care is delivered is becoming much more urgent. There are growing pressures from changes in medicine, a shrinking workforce, and increasing demands made on health care. Small incremental changes will not be enough to deal with these pressures. Could we harness the innovation, discipline, ability to borrow from elsewhere, and willingness to challenge the status quo that has often typified the quality movement and the best of medicine? Can we scale up the methodology of quality improvement to help those planning parts of the wider healthcare system do it better? I suggest three ways that this might be done.

\section{CHALLENGING ASSUMPTIONS}

Perhaps the area where quality improvement methods could have the most impact is in helping to challenge the assumptions and preconceptions about how health systems work using the key insight that every system is perfectly designed to achieve the result it gets. Many of the assumptions used as the basis for planning are often based on past custom rather than evidence. Nevertheless, they have often been elevated to the status of immutable rules. Many of the most dysfunctional aspects of current systems are a direct consequence of problems with these underlying assumptions and design principles.

The quality improvement discipline of rigorously identifying objectives and designing the service to achieve them is as relevant to large scale systems as it is to a single clinical intervention. An example of this approach at a national level is the Institute of Medicine's Crossing the quality chasm report on healthcare in the USA. ${ }^{1}$ Examples of the Institute of Medicine's call to change the rules included moving from care based on encounters to one based on relationships, from information as a record to knowledge being shared and information flowing freely, moving from secrecy to transparency, reaction to anticipation, and from focusing on cost reduction to eliminating waste.

All countries will have assumptions that need to be challenged. In the UK one of the strangest is the division between primary and secondary care, which, in the way it is constructed, seems to have more to do with the medical politics of the 1940s than the appropriate division of labour between different areas of expertise. Hospitals perform large amounts of primary care and a significant amount of secondary care happens outside hospital. A related assumption is that specialists usually work for and at one institution and do so for most of their career. Some specialists in the UK are now working as part of wider networks and are finding that this makes better use of their expertise, improves care, and gives greater peer support. Other countries have their own shibboleths and it is likely that they have the same effect as in the UK; to constrain thinking, prevent innovative solutions from emerging and protect the status quo-however unviable.

\section{UNDERSTANDING HOW PROCESSES WORK}

The techniques of systems thinking used in quality improvement, for example in areas such as process mapping, need to be better applied to the planning of healthcare delivery. Too often planning uses modelling approaches that fail to understand the importance of variability, feedback loops, and human behaviour. For example, in Israel, the UK, and elsewhere, this has led to hospital planners assuming occupancy levels in excess of $90 \%$ will be possible without any adverse effects on efficiency or patients' experience. Understanding the variability of demand quickly confirms what experience shows; that this is impossible and the results are undesirable. These insights would lead to planners replacing design principles that value "sweating the assets" with others based on safety, eliminating waiting, improving the patient experience, and ensuring that patients flow through the hospital rather than being subject to a regime of "hurry up and wait". This could make a major difference to the way hospitals and other services work and the design of new facilities.

\section{SEEING THROUGH THE PATIENTS' EYES}

Can we honestly say that the quality improvement discipline of seeing through the patient's eyes has been sufficiently widely used to plan healthcare delivery? These insights are used to improve the quality of patients' interactions with clinicians and their experience of care. But at the level above this, in the planning of hospitals and the systems they are part of, the use of this technique is much less common. If this approach were more widely applied new models for service delivery might emerge including using more nonhealth care settings for routine care, using information technology to deliver care in patients' homes, and making patients the designers and co-producers of care.

\section{CONCLUSION}

The discipline and methods of quality improvement could help planners and policy makers think differently about how to improve the design of healthcare buildings and systems. It is important that this happens as there is little point in improving front line clinical delivery if it is embedded in a wider system that is dysfunctional. Quality improvement methods offer the chance to find innovative ways to solve some of the most intractable problems facing many healthcare systems by providing an approach that challenges assumptions, tests new models, and ensures that services meet patients' needs. Without new ways of tackling these issues health services will continue to disappoint an increasingly demanding public and wear out the patience of those who pay the bills

Qual Saf Health Care 2005; 14:75.

doi: 10.1136/qshc.2005.013748

Correspondence to: N Edwards, Policy

Director, NHS Confederation, UK:

nigel.edwards@nhsconfed.org

\section{REFERENCES}

1 Institute of Medicine. Crossing the quality chasm: a new health system for the 21 st Century. Washington DC: National Academy Press, 2001. 